\title{
36. THEORY OF STELLAR ATMOSPHERES (THEORIE DES ATMOSPHERES STELLAIRES)
}

PRESIDENT: W. Kalkofen

VICE PRESIDENT: L. E. Cram

ORGANIZING COMMITTEE: Y. Cuny, D. Dravins, D. F. Gray, J. L. Linsky, R. Pallavicini, A. Peraiah, A. Sapar, M. J. Seaton, T. Takashi, R. Wehrse, L. A. Willson, C. Zwaan

Several members of the Organizing Committee as well as Dr. Lee Hartmann have written in their area of expertise for the Report of Commission 36 to the IAU. Their contributions'are assembled here with minimal editing.

The topics treated in this report are:

Magnetic Activity in Stars (R. Pallavicini)

Stellar Activity Cycles (D. F. Gray)

Developments in Stellar Coronae (J. L. Linsky)

Dynamical Atmospheres and Winds (L. A. Willson)

Disk and Envelope Emission from Young Stellar Objects (L. Hartmann)

The Atmospheric Structure of Very Cool Stars (T. Tsuji)

Multidimensional Radiative Transfer (R. Wehrse)

Some of the individual reports make extensive reference to review papers and conference proceedings. To these should be added IAU Colloquium No 143 on The Sun as a Variable Star, the first IAU Colloquium specifically addressed to the so-called solar-stellar connection, the proceedings of which are to be published by Cambridge University Press and by Kluwer.

Below are the individual reports.

\section{MAGNETIC ACTIVITY IN STARS (Roberto Pallavicini)}

\section{General Overview}

Over the past decade it has become increasingly evident that magnetic fields play a fundamental role in the outer atmosphere of cool stars. It is not only that magnetic fields are directly responsible for solar-type activity phenomena like spots, plages and flares, but they also determine in a significant, perhaps dominant, way the structuring, energy balance and dynamics of the outer atmospheric layers. This new perspective in stellar atmospheric research has largely been a consequence of X-ray and UV observations from space (particularly with the Einstein and IUE satellites), as well as of new observational techniques, of vastly improved sensitivity and spectral resolution, developed for ground based optical and radio work. The new data have demonstrated the occurrence on stars of magnetic phenomena similar to those observable on the Sun, and have prompted the development of the so-called "solar-stellar connection", i.e. the close interaction of solar and stellar studies to the benefit of both disciplines.

In the last three years, research on stellar magnetic activity has continued to be actively pursued, as amply demonstrated by the many conferences, review articles and books which have been devoted to the subject. The number of research papers that have appeared in the major scientific journals is so large that it would be impossible to summarize them here. Rather, the emphasis will be placed on review articles and conference proceedings that present an up-to-date overview of the field. The reader is referred to these reviews for further details and complete bibliographical lists. It is also important to mention that the surveyed period has witnessed the start of operations of ROSAT, the Hubble Space Telescope and the EUVE satellite. These space missions, together with the continuing operations of IUE, and the availability of high quality photometric and spectroscopic optical data, are contributing enormously to our understanding of magnetic activity in stars.

\section{Conferences and books}

Several conferences have been devoted to stellar magnetic activity (or to specific aspects of it) over the past three years. It is interesting to note that the majority of them were dedicated to 
the joint discussion of solar and stellar phenomena, in an effort to bring together researchers from two communities that were originally virtually separated. A good example is the conference on Mechanisms of Chromospheric and Coronal Heating (CH91) held in Heidelberg on 5-8 June 1990. Other examples are the IAU Joint Commission Meeting on Solar and Stellar Coronae (SC92) held in Buenos Aires on 27 July 1991, and the G.S. Vaiana Memorial Symposium Advances in Stellar and Solar Coronal Physics (SC93) held in Palermo on 22-26 June 1992. Along the same lines are also the Seventh Cambridge Workshop on Cool Stars, Stellar Systems and the Sun (CS92) held in Tucson on October 9-12, 1991, and the IAU Colloquium No. 130 The Sun and Cool Stars: Activity, Magnetism, Dynamos (MD91), held in Helsinki on 17-20 July 1990. The proceedings of these meetings offer a unique opportunity to compare observational results and theoretical models for both the Sun and other cool stars.

Other more specialized meetings were the Armagh Bicentennial Colloquium on Surface Inhomogeneities on Late-Type Stars (SI92) held in Armagh on 24-27 July 1990, and the NATO ASI on Angular Momentum Evolution in Young Stars (AM91) held in Noto on 17-21 September 1990. General reviews on magnetic activity in stars have been contributed by Zwaan (CH91), Rosner (HM91), Rodonò (AM91 and SI92), Baliunas (1991), Radick (1991), Feigelson, Giampapa and Vrba (1991), Montmerle (SC92), Pallavicini (1992a,b), Schrijver (CH91 and 1993) and Catalano (SC93). A catalog of chromospherically active binary stars, which updates an early version of 1988 , has been published by Strassmeier et al. (1993).

\section{Research highlights}

\section{a) Rotation, differential rotation, and dynamo action}

In stars with outer convective zones (i.e. $\sim$ F0 or later), surface magnetic fields are believed to be produced by a dynamo mechanism that involves the interaction of rotation and turbulent convection. The present status of dynamo theory is discussed in MD91. As stressed by Brandenburg $\&$ Tuominen in those proceedings, the $\alpha \Omega$-dynamo can reproduce the general features of the solar cycle, but a completely satisfactory theory is still lacking. More important, the fact that many free parameters can be adjusted to fit one single object (the Sun !) does not provide a conclusive test of the theory. It is not even clear whether all types of stars should have the same dynamo pattern as the Sun. Theoretical arguments would rather suggest the opposite (Weiss SC93) and observational data exist that indicate a different behaviour including the existence of polar spots and of stars rotating more rapidly at the poles than at the equator (Eaton SI92; Vogt \& Hatzes MD91). Recent advances in the study of stellar rotation, and its consequences on dynamo activity, have been reviewed by Bouvier (AM91) for pre-main sequence stars, by Stauffer (AM91) and Soderblom (AM91) for main-sequence stars, and by Gray (AM91) for giants. A "rotostat" mechanism has been proposed by Gray (AM91) to explain magnetic activity and rotational evolution in evolved stars. Among the cooler giants and supergiants, magnetic fields may play only a negligible role (Dupree HM91, Cuntz \& Stencel HM91). Rosner (SC93) has discussed the problem of dividing lines in the HR diagram separating stars with magnetically confined coronae from stars with no coronae and large mass loss rates.

\section{b) Measurements of stellar magnetic fields}

It has become possible in recent years to measure photospheric magnetic fields in cool stars from the Zeeman broadening of magnetically sensitive lines. Since the observations are made by using unpolarized light, the intrinsic difficulty of opposite polarities cancelling each other in complex magnetic field topologies is eliminated. Recent advances in this field, including the discussion of possible systematic errors, can be found in Saar (MD91) and Solanki (CS92). The observations have provided magnetic field strengths and filling factors for a large number of cool stars, including some dMe flare stars and one pre-main sequence object. The inferred magnetic field refers to an average over the star surface. Instead, there is virtually no information on the magnetic field strength in localized active regions such as spots and plages. These magnetic field measurements are possible only for slowly rotating stars, since it is difficult to separate the small Zeeman broadening from rotational broadening in rapidly rotating stars. A technique has been developed to overcome this difficulty, by separating the contribution of magnetic regions in velocity ("Zeeman Doppler imaging"). Measurements of magnetic fields of hotter Ap stars have 
been reviewed by Landstreet (1992). Their magnetic field is much simpler than in cooler stars, with an overall structure that is roughly dipolar and extends over virtually the entire star.

\section{c) Non-radiative heating}

The long-standing problem of non-radiative heating in stellar chromospheres and coronae is far from solved (see $\mathrm{CH} 91$ ). The observational evidence points at the dominant role of magnetic fields, except perhaps for the quiet chromosphere that is more likely heated by acoustic waves (Ulmschneider CH91). MHD waves and/or electric currents are certainly required to heat the coronae of cool stars, but there is no general consensus on the detailed mechanism. The new $\mathrm{X}$-ray data on stellar coronae made available by ROSAT (Schmitt CS92, SC93; Bromage CS92, SC93) confirm and extend previous results from Einstein (Vaiana et al. SC92, Sciortino SC93), but are unlikely to provide a definite answer to the heating problem. Clues could more easily be provided by spatially resolved observations of the Sun. Magnetic effects are also required to explain most of the observed chromospheric emission, particularly in active stars whose integrated flux is dominated by the contribution of magnetic regions. However, the so called "basal flux" in the $\mathrm{Ca}$ II and $\mathrm{Mg}$ II lines (Zwaan CH91, Schrijver CH91) may result from acoustic heating.

\section{d) Photometric and spectroscopic observations of starspots}

The presence of dark cool spots on the surface of many active stars (of the RS CVn and BY Dra classes) can be inferred from photometric variations, accompanied by color changes that indicate that the star is redder at the photometric minimum. In principle, the systematic monitoring of photometric changes permits to infer the spot size and location, and the variations of stellar rotation with latitude (differential rotation). In practice, things are much more complicated, and one has to worry about the uniqueness of the solution and systematic effects (Eaton SI92, Hall MD91). An alternative way to map surface inhomogeneities is to use spectroscopic observations, and the subtle changes induced by spots on a rotationally broadened line profile ("Doppler imaging"; Piskunov MD91, Vogt \& Hatzes MD91, Collier Cameron S192). This technique is starting to provide realistic maps of the otherwise unresolvable stellar disks. The existence of polar spots -that have been inferred from both Doppler imaging and analysis of photometric light curves- is however still controversial (Byrne SI92).

\section{e) Chromospheric and coronal magnetic features}

The presence of chromospheric plages and coronal active regions on stars can be inferred indirectly from rotational modulation of chromospheric and coronal emission and/or from the comparison of stars of similar spectral type, but very different activity levels (see reviews by Baliunas 1991, Pallavicini 1992a, Catalano SC93). Observations in the lines of $\mathrm{Ca} \mathrm{II} \mathrm{and} \mathrm{Mg} \mathrm{II}$, as well as in UV lines and X-rays, show in fact a wide range of emission levels at each effective temperature. This is interpreted as being due to different fractions of the star surface covered by magnetic (plage) regions. Multi-component modelling of $\mathrm{Mg}$ II line profiles have been used to map the distribution of these regions on the stellar surface at different phases (Neff SI92). Similarly, rotational modulation and eclipses have been used in X-rays to infer the spatial structure of stellar coronae (Schmitt CS92, Foing SC93). The variability of coronal emission as produced by surface magnetic activity has been reviewed by Cheng \& Pallavicini (SC92) and Pallavicini (SC93). The correlation between chromospheric/coronal emission and photometric variations (spots) is usually not very good, probably because of the high variability of the former on short time scales (Byrne SI92, Linsky SI92).

\section{f) Stellar flares}

Observations of stellar flares have been reviewed recently by Haisch, Strong and Rodonò (1991), Pallavicini (1992b) and Byrne (SC93). There is a strong similarity between solar flares and the typically more energetic flares that occur on dMe flare stars and RS CVn binaries (and occasionally also on other types of cool stars). This suggests that the process of energy release is basically the same in the two cases. Theoretical models of flares in stars and accretion disks have been discussed by Kuijpers (1992). A comprehensive summary of recent observations and empirical models of stellar flares is given in a book edited by Pettersen (1991) to which we refer the reader 
for details on the subject.

\section{References}

CH91: Mechanisms of Chromospheric and Coronal Heating, P. Ulmschneider, E.R. Priest, and R. Rosner (Eds.), 1991, Springer-Verlag: Berlin.

MD91: The Sun and Cool Stars: Activity, Magnetism, Dynamos, I. Tuominen, D. Moss, G. Rüdiger (Eds.): 1991, Springer-Verlag: Berlin.

AM91: Angular Momentum Evolution of Young Stars, S. Catalano, and J.R. Stauffer (Eds.), 1991, Kluwer: Dordrecht.

SI92: Surface Inhomogeneities on Late-Type Stars, P.B. Byrne, and D.J. Mullan (Eds.), 1992, Springer-Verlag: Berlin.

SC92: Solar and Stellar Coronae, R. Pallavicini (Ed.), 1992, Memorie Soc. Astron. Ital. 64, No. 3-4.

CS92: Seventh Cambridge Workshop on Cool Stars, Stellar Systems, and the Sun, M.S. Giampapa, and J.A. Bookbinder (Eds.), 1992, Astron. Soc. Pacific: San Francisco.

SC93: Physics of Solar and Stellar Coronae: G.S. Vaiana Memorial Symposium, J.A. Linsky, and S. Serio (Eds.), 1993, Kluwer: Dordrecht.

Baliunas, S.: 1991, in The Sun in Time (Sonett, C.P., Giampapa, M.S., and Matthews, M.S. eds.), University of Arizona: Tucson. p. 809.

Feigelson, E.D., Giampapa, M.S., and Vrba, F.J.: 1991, in The Sun in Time (Sonett, C.P., Giampapa, M.S., and Matthews, M.S. eds.), University of Arizona: Tucson, p. 658.

Haisch, B.M., Strong, K.T., and Rodonò, M.: 1991, Ann. Rev. Astron. Ap. $29,275$.

Kuijpers, J.: 1993, in The Sun: A Laboratory for Astrophysics (J.T. Schmeltz and J.C. Brown eds.), Kluwer: Dordrecht, p. 535.

Landstreet, J.D.: 1992, Ann. Rev. Astron. Ap. 4, 35.

Pallavicini, R.: 1992a,b, in The Sun: A Laboratory for Astrophysics (J.T. Schmeltz and J.C. Brown eds.), Kluwer: Dordrecht, pp. 313 and 509.

Pettersen, B.R. (Ed.): 1991, Stellar Flares, special issue of Memorie Soc. Astron. Ital., Vol. 62, No. 2.

Radick, R.R.: 1991, in The Sun in Time (Sonett, C.P., Giampapa, M.S., and Matthews, M.S. eds.), University of Arizona: Tucson, p. 787.

Schrijver, C.J.: 1993, in Inside the Stars, proceedings of IAU Colloquium No. 137, Vienna: April 1992.

Strassmeier, K.G., Hall, D.S., Fekel, F.C., and Scheck, M.: 1993, Astron. Astrophys. Suppl. 100, 173.

\section{STELLAR ACTIVITY CYCLES (David F. Gray)}

No attempt is made to be complete or exhaustive in citing references. Those cited are examples or reviews, and are meant simply as starting points for the interested reader.

Changes in Stellar Atmospheres During Magnetic Cycles. The dynamics of stellar atmospheres for stars on the cool half of the H-R diagram present real challenges for the inventive researcher.

The change in the solar irradiance by $0.1 \%$ during the last solar cycle has enlivened the interest in stellar magnetic cycles and the atmospheric changes that take place. Two competing effects of the magnetic field are apparent from the solar observations: local inhibition of convection causing reduction in irradiance when magnetic fields are strong, as exemplified in sunspots, and a more global enhancement of irradiance through the maximum of the magnetic cycle (Fröhlich et al. 1991, Pap 1994, Foukal \& Lean 1990). The time scales for the effects of spots are the spot lifetimes and the rotational modulation, both of which are weeks. The time scale of the global enhancement is the eleven years or so associated with the solar cycle.

Photometric and spectroscopic variations of stars arising from spots have been known for some time, and cycle-type variations in chromospheric emission of stars has also been well documented (Eaton et al. 1993, Jankov \& Foing 1991, Byrne \& Mullan 1991, Radick et al. 1990, Gray 
1988). More recently, variations in temperature and granulation and photometric variations have been observed on the many-year time scale. Not all stars show variations, but for those that do, the changes in chromospheric emission occur before those in temperature by an amount that appears to depend on spectral type, ranging from three years for early $G$ to near zero for early $\mathrm{K}$ dwarves. Photometric changes lie in between, and granulation changes seem to be in phase with the temperature changes (Gray et al. 1993 \& forthcoming papers; Gray 1994). Temperature changes during the solar cycle have yet to be measured (Livingston \& Steffen 1988). A clever satellite experiment to measure variations in the solar radius is currently in progress (Sofia 1994, see also Ribes et al. 1991).

It is not clear from the solar observations alone whether the 11-year variation in solar irradiance indicates a real variation in power output or a change in angular distribution of the solar radiation (Kuhn 1991). But since photometric and temperature variations are now being seen for stars, where the orientation of the rotation axes is random, it seems likely that real power variations are occurring. In either case, ramifications for terrestrial climate may be important (Baliunas \& Jastrow 1993).

Evidence for differential rotation in a Com (HD 114710, G0 V) has been extracted from the Mt. Wilson HK observations (Donahue \& Baliunas 1992). If confirmed, dynamo theorists will have something more to chew on since this star's equator rotates more slowly than do the higher latitudes, the opposite of the solar example.

\section{Radial Velocity Variations}

Several precision radial velocity programs are detecting periodic variations, especially in evolved stars. Larson et al. (1993) find a $46 \mathrm{~m} / \mathrm{s}$ variation with a period of 585 days for a Gem (Ko III), while Smith et al. (1987) detected $160 \mathrm{~m} / \mathrm{s}$ variations with a 1.84 day period in 'Boo (K2 III). The source of these variations is not yet clear, but possible explanations include rotational modulation arising from surface features, oscillations, and the surfacing of large convective cells. But stars nearer the main sequence, such as ' $\mathrm{CMi}$ (F5 IV-V) also show intrinsic variable radial velocity of a few $\mathrm{m} / \mathrm{s}$ (Cochran \& Hatzes 1993), although these may be low-amplitude k-Scuti type oscillations.

\section{Hydrodynamical Computations}

Numerical and analytical modeling from basic equations is being carried out by a number of groups. Propagation and confinement of MHD waves has been studied by Rosner et al. (1991). The mechanisms involved in the formation and heating of coronal loops has been investigated by Ulmschneider et al. (1991) and Lou (1992). Granulation and convective structure have been computed by Hossain \& Mullan (1991), Cataneo \& Malagoli (1992), and Stein et al. (1992), giving passably good agreement with the observations and physical insight into convective structure well below the surface. An excellent review of the solar observations of granulation is given by Spruit et al. (1990).

Progress on a physical explanation of the granulation boundary (Gray \& Nagel 1989) has been made by Kim and Fox (1992).

\section{References}

Baliunas, S.L. \& R. Jastrow 1993. Energy: The International Journal, in print.

Byrne, P., \& D. Mullan (eds.) 1991. Surface Inhomogeneities in Late-Type Stars, (Springer: Berlin)

Cattaneo, F. \& A. Malagoli 1992. Seventh Cambridge Worshop on Cool Stars, Stellar Systems, and the Sun, (Ast. Soc. Pacific: San Francisco), M.S. Giampapa \& J.A. Bookbinder, eds., p. 139.

Cochran, W.D. \& A.P. Hatzes 1993. Ap. Space Sci. in print.

Donahue, R.A. \& S.L. Baliunas 1992. Ap.J. 393, L63.

Eaton, J.A., G.W. Henry, C. Bell, \& A. Okorogu 1993. Astron. J., submitted.

Foukal, P. \& J. Lean 1990. Science 247, 504.

Fröhlich, C., P.V. Foukal, J.R. Hickey, H.S. Hudson, \& R.C. Wilson 1991. The Sun in Time, 
(Univ. Arizona: Tucson), C.P. Sonett, M.S. Giampapa, M.S. Mathews, eds., p. 11.

Gray, D.F. 1988. Lectures on Spectral-Line Analysis: F, G, and K Stars, (The Publisher: Arva, Ontario).

Gray, D.F. 1994. Publ. Astron. Soc. Pacific, submitted.

Gray, D.F., S.L. Baliunas, G.W. Lockwood, \& B.A. Skiff 1993. Ap.J. 400, 681.

Gray, D.F. \& T. Nagel 1988. Ap.J., 341, 421-426.

Hossain M. \& D.J. Mullan 1991. Ap.J. 380, 631.

Jankov, S. \& B.H. Foing 1992. Astron. Ap. 256, 533.

Kim, Y.-C. \& P.A. Fox 1992. Seventh Cambridge Worshop on Cool Stars, Stellar Systems, and the Sun (Ast. Soc. Pacific: San Francisco), M.S. Giampapa \& J.A. Bookbinder eds., 172.

Kuhn, J.R. 1991. Adv. Space Res. 11, (4)171.

Larson, A.M., A.W. Irwin, S.L.S. Yang, C. Goodenough, G.A.H. Walker, A.R. Walker, \& D.A. Bohlender 1993. Publ. Astron. Soc. Pacific, submitted.

Livingston, W. \& M. Steffen 1988. Adv. Space Res. 8, 133.

Lou, Y.Q. 1992. Ap.J. 395, 682.

Pap, J.M. (ed.) 1994. The Sun as a Variable Star: Solar and Stellar Irradiance Variations, (Cambridge: Cambridge).

Radick, R.R., G.W. Lockwood, \& S.L. Baliunas 1990. Science 247, 39.

Ribes, E., B. Beardsley, T.M. Brown, Ph. DeLanche, F. Laclare, J.R. Kuhn, \& N.V. Leister 1991. The Sun in Time, (Univ. Arizona: Tucson), C.P. Sonett, M.S. Giampapa, M.S. Mathews, eds., 59 .

Rosner, R., C.-H. An, Z.E. Musielak, R.L. Moore, \& S.T. Suess 1991. Ap.J. Lett. 372, L91.

Smith, P.H., R.S. McMillan, \& W.J. Merline 1987. Ap.J. 317, L79.

Sofia, S. 1994. in The Sun as a Variable Star: Solar and Stellar Irradiance Varia-tions, (Cambridge: Cambridge).

Spruit, H.C., . Nordlund, \& A.M. Title 1990. Ann. Rev. Ast. Ap. 28, 263.

Stein, R.F., A. Brandenburg, \& . Nordlund 1992. Seventh Cambridge Worshop on Cool Stars, Stellar Systems, and the Sun, (Ast. Soc. Pacific: San Francisco), M.S. Giampapa \& J.A. Bookbinder, eds., 148.

Ulmschneider, P., K. Zähringer, \& Z:E. Musielak 1991, Astron. Ap. 106, 9.

\section{DEVELOPMENTS IN STELLAR CORONAE (Jeffrey L. Linsky)}

This report summarizes the major developments (observational and theoretical) in the study of stellar coronae driven in large part by the analysis of the new data sets from ROSAT, EUVE, ASCA, Yohkoh, and HST, together with the continuing analysis of Einstein data and radio observations.

\section{Introduction}

During the past three years new and more powerful telescopes have allowed observers to study stellar coronae with higher energy resolution, observe fainter $\mathrm{X}$-ray sources, observe large numbers of stars in the unexplored EUV region (80-700 $\AA$ ), and survey the entire sky in $x$-rays and the EUV. These important capabilities result primarily from the new ROSAT, EUVE, and ASCA satellites. At the same time the Einstein archive has been mined further with the distribution of its archive on CDRoms, and the VLA, the new Australian Telescope, and VLBI networks are studying coronae at radio wavelengths. The spectacular Yohkoh $\mathrm{x}$-ray solar images provide vivid evidence that the solar corona (and by implication stellar coronae) is highly dynamic with the heating and expansion of magnetic loops on many time scales (see papers in the 1992 October issue of Publ. Astr. Soc. Japan and [25]).

Launched on 1990 May 31, the Roentgensatellit (ROSAT) [46] includes two x-ray imaging detectors (the PSPC [37] and the HRI covering 0.1-2.4 keV) and the Wide Field Camera (WFC) for broadband imaging in the 60-200 $\AA$ region [38]. The all sky survey portion of the mission (August 1990 to February 1991) provided the first deep survey of the x-ray (see review by Schmitt in [25]) and EUV sky down to faint limits. Of the 384 sources listed in the WFC Bright Source Catalog [38] 
about $50 \%$ are known to be nondegenerate stars. Launched on 1992 June 7, EUVE [27] obtained broadband EUV fluxes in the 70-760 $\AA$ region during its all sky survey with about 350 stars listed in the initial catalog [28], and is obtaining EUV spectra and broadband fluxes during its pointed phase. A number of early papers based on EUVE data are in the 1993 September 10 issue of Ap.J. Letters. ASCA has been obtaining x-ray observations of stars with higher energy resolution and broader range (to $12 \mathrm{keV}$ ) than ROSAT since 1993 February 20. The G.S. Vaiana Memorial Symposium [25] contains a number of important review papers, including summaries of the ROSAT and Yohkoh work as of June 1992. For earlier comprehensive reviews of stellar coronae see [33] and papers in the proceedings of two symposia [12,17]. A recent comprehensive review of radio emission from stellar coronae is found in [19] and in the contribution by Drake in [25].

\section{Late-Type Stars}

Coronae are probably ubiquitous in late-type dwarfs and at least G-type giants as indicated by $\mathrm{x}$ ray, radio, and EUV emission, and most recently by the detection with HST/GHRS of the Fe XXI $1354 \AA$ line in the M dwarf AU Mic [29]. A ROSAT survey of very late M dwarfs [21] shows that the coolest $M$ dwarfs, which have fully convective interiors, are just as $x$-ray bright as the early $M$ dwarfs in which solar-type dynamos operate. Coordinated ROSAT and VLA observations of $M$ dwarfs [21] show the good correlation of $x$-ray and radio luminosities [20]. An x-ray survey of late-type giants and supergiants from the Einstein archive shows many detections of $\mathrm{F}$ and $K$ giants and confirms the existence of the $x$-ray dividing line located at middle- $K$ spectral type [26]. The ROSAT all sky survey data [22] support this result. A WFC survey of nondegenerate stars within $10 \mathrm{pc}$ detected 41/220 mostly F-M dwarfs [51], and Hodgkin and Pye [25] present a WFC survey of stars extending out to $25 \mathrm{pc}$. Hybrid-chromosphere stars are now detected as $\mathrm{x}$-ray sources indicating that they have coronae $[4,39]$.

Analysis of Einstein IPC archival data of the Pleiades [31] and Ursa Major [41] clusters provide new information on the age and rotational velocity dependence of coronal $\mathrm{x}$-ray emission. The first survey of the Hyades by ROSAT has been published [45] and surveys of other clusters are under way [25].

$M$ dwarf stars and especially $\mathrm{dMe}$ stars are bright, transient $\mathrm{x}$-ray [35,23] and radio [3] emitters during flares, indicating large enhancements in the thermal and nonthermal electrons in their coronae. For a review of stellar flares see [34]. VLBI measurements have now resolved the radio emission from dMe stars [2], providing direct estimates of the brightness temperatures. Coordinated multiwavelength observations such as the RIASS campaign (ROSAT and IUE) [1] are needed to study quiescent and flaring properties of the corona and lower atmosphere together.

\section{Binary Systems with Nondegenerate Components}

Binary systems containing nondegenerate components that have hot coronae as indicated by their luminous x-ray and radio emission include the RS CVn, BY Dra, W UMa, and Algol systems. The ROSAT PSPC all sky survey contains $136 \mathrm{x}$-ray detected RS CVn systems [6] which can be modelled with a two-temperature coronal plasma at $\log \mathrm{T}=6.3$ and 7.2 [7]. There is an excellent correlation between the radio and Einstein IPC fluxes for a very large sample of RS CVn systems [8], despite the usual interpretation of the $\mathrm{x}$-ray emission as thermal and radio emission as nonthermal [30]. Coordinated ROSAT, IUE, and VLA observations during the all-sky survey [16] confirm the earlier nonsimultaneous data results. The remarkable radio-x-ray correlation, which extends over five orders of magnitude and includes many classes of late-type stars [20], probably indicates that heating and particle acceleration occur in parallel. A systematic survey of the radio emission from classical Algol systems [48] shows that they have properties similar to the RS CVns, whereas the radio emission from young Algol-type systems (Serpentids) is probably from their winds [11]. A two-dimensional model of the magnetospheres of active late-type stars [32] explains the quiescent radio emission as gyrosynchrotron emission from trapped relativistic electrons, and time-dependent models of the nonthermal particle populations and the resultant radio emission are now available [5]. The first three-dimensional picture of a stellar corona has been constructed [43] from EXOSAT observations of AR Lac. Active binary systems are known to flare as seen in $x$-rays $[23,10]$ and radio emission [3]. 


\section{Early-Type Stars}

The usual explanation for the luminous $x$-ray emission from O-type and early B-type stars is shock-heated gas in their winds (see review by Kudritzki and Hillier in [25]) rather than coronae, but an alternative corona-like model has been proposed [49] with magnetic plasma heated in current sheets. The Einstein IPC archive contains 74 early B-type stars that are $\mathrm{x}$-ray sources with properties similar to the 0 -type stars and $34 / 1058$ late-B stars, indicating that $\mathrm{x}$-ray emission from these stars is either rare or from a late-type companion [18]. The ROSAT HRI and PSPC detected young late-B type stars, but the spread in $L_{x}$ is large [36] and the origin of the $x$-ray emission remains a puzzle [42]. The Einstein archive data [41] and new ROSAT PSPC observations [44] contain very few $x$-ray detected single A-type stars and show a systematic decline in coronal x-ray brightness with increasing $T_{\text {eff }}$ that may be due to strong coronal winds [44]. The magnetic chemically peculiar stars of both the helium-strong (early-B) and helium-weak (late-B) types are both radio [24] and $x$-ray sources [9]. The radio data may be explained by a magnetospheric model in which the stellar wind distends the magnetic field lines leading to gyrosynchrotron emission by relativistic electrons [24].

\section{Theoretical and Spectroscopic Work}

Analysis of the x-ray and EUV radiation requires accurate representations of the coronal plasma emissivities. Reviews of $x$-ray spectroscopy and the emissivity of stellar coronae can be found in [17]. For a recent summary of the important spectral lines in the 50-500 $\AA$ range and densitysensitive line ratios based on studies of the solar corona see [14]. One major complication in the analysis of stellar $x$-ray spectra is that solar (and by implication stellar) coronal abundances differ from those of the photosphere [13].

Analysis of low resolution x-ray stellar spectra obtained with the Einstein SSS and the EXOSAT TGS have been modelled with two-temperature coronae. Einstein IPC observations of 130 latetype stars can also be modelled with plasmas at two temperatures, including very hot coronae $\left(T>10^{7.5} \mathrm{~K}\right)$ of RS CVn systems, but models with continuous emission measure distributions also fit the data and are physically; more plausible [40]. A combined analysis of both the EUV and x-ray fluxes of many late-type dwarfs [50] leads to an upward revision of the temperatures in two-temperature coronal models by a factor of 2 to $\log \mathrm{T}=6.7$ and 7.5 . This may explain why the WFC has detected fewer M dwarfs than predicted on the basis of the earlier temperature estimates [38]. Higher resolution spectra from EUVE, ASCA, and future spacecraft are needed to test the validity of two-temperature coronal models. For a comprehensive summary of the energy budget requirements and theoretical mechanisms of chromospheric and coronal heating see [47]. Recent reviews of coronal heating mechanisms include those by Priest and Rosner in [25].

\section{References}

[1] Ayres, T.R., et al. 1994, submitted to ApJ.

2. Benz, A.O. and Alef, W. 1991, Astr. Ap. 252, L19.

3. Bookbinder, J.A. 1991, Mem S.A.It 62, 321.

4. Brown, A., et al. 1991, Ap. J. 373, 614.

5. Chiuderi-Drago, F. and Franciosini, E. 1993, ApJ, in press.

6 Dempsey, R.C., Linsky, J.L., Fleming, T.A., and Schmitt, J.H.M.M., 1993a, A p. J. S.86, 599.

7 Dempsey, R.C., Linsky, J.L., Schmitt, J.H.M.M., and Fleming, T.A., 1993b, Ap. J. 413, 333.

8. Drake, S.A., Simon, T., and Linsky, J.L. 1992, Ap. J. S.82, 311.

9. Drake, S.A., et al. 1993, ApJ, in press.

[10] Doyle, J.G., et al. 1991, M.N.R.A.S. 248, 503.

11 Elias, N.M. and Güdel, M. 1993, A. J.106, 337.

[12] Elvis, M. 1990, Imaging X-ray Astronomy: A Decade of Einstein Observatory Achievements (Cambridge: Cambridge U. Press).

[13] Feldman, U. 1992, Physica Scripta 46, 202.

14 Feldman, U., et al. 1992, Ap. J. S.81, 387.

15 Fleming, T.A., et al. 1993, Ap. J. 410, 387.

16 Fox, D., et al. 1994, A\&A, in press.

17] Gorenstein, P and Zombeck, M. 1990, High Resolution X-ray Spectroscopy of Cosmic Plasmas (Cambridge: Cambridge U. Press). 
[18] Grillo, F., et al. 1992, Ap. J. S.81, 795.

19 Güdel, M. 1994, ApJS, in press.

20 Güdel, M. and Benz, A.O. 1993, Ap. J. (Letters) 405, L63.

21 Güdel, M., et al. 1993, ApJ, in press.

22 Haisch, B., et al. 1991, Ap. J. (Letters) 383, L15.

23 Linsky, J.L. 1991, Mem S.A.It 62, 307.

24 Linsky, J.L., Drake, S.A., and Bastian, T.S. 1992, Ap. J. 393, 341.

25] Linsky, J.L. and Serio, S. 1993, Physics of Solar and Stellar Coronae: G.S. Vaiana Memorial Symposium (Dordrecht: Kluwer).

[26] Maggio, A., et al. 1990, Ap. J. 348, 253.

27) Malina, R.F. and Bowyer, S. 1991, in Extreme Ultraviolet Astronomy, ed. R.F. Malina and S. Bowyer (New York: Pergamon), 397.

[28] Malina, R. et al. 1993, ApJ, in press.

29. Maran, S.P., et al. 1994, ApJ, in press.

30. Massi, M. and Chiuderi-Drago, F. 1991, Astr. Ap. 253, 403.

31 Micela, G., et al. 1990, Ap. J. 348, 557.

32 Morris, D.H., Mutel, R,L., and Su, B. 1990, Ap. J. 362, 299.

33. Pallavicini, R. 1989, A\&A Reviews 1, 177.

34] Pallavicini, R. 1991, in The Sun: a Laboratory for Astrophysics, ed. J.C. Brown and J.T. Schmelz.

[35] Pallavicini, R., Tagliaferri, G., and Stella, L. 1990, Astr. Ap. 228, 403.

36. Patten, B.M. and Simon, T. 1993, to appear in ApJL.

37. Pfeffermann, E., et al. 1987, in Soft X-ray Optics and Technology, Proc. SPIE 733, 519.

38. Pounds, et al. 1993, M.N.R.A.S. 260, 77.

39 Reimers, D. and Schmitt, J.H.M.M. 1992, Ap. J. (Letters) 392, L55.

40 Schmitt, J.H.M.M., et al. 1990a, Ap. J. 365, 704.

41 Schmitt, J.H.M.M., et al. 1990b, Ap. J. 351, 492.

42 Schmitt, J.H.M.M., et al. 1993, Ap. J. (Letters) 402, L13.

43. Siarkowski, M. 1992, MNRAS 259, 453.

44 Simon, T. and Drake, S.A. 1993, to appear in AJ.

45 Stern, R.A., et al. 1992, Ap. J. (Letters) 399, L159.

46 Trümper, J., et al. 1983, Nature 349, 579.

[47] Ulmschneider, P., Priest, E.R., and Rosner, R. 1990, Mechanisms of Chromospheric and Coronal Heating (Berlin: Springer-Verlag).

[48] Umana, G., Catalano, S., and Rodonò, M. 1991, Astr. Ap. 249, 217.

49 Usov, V.V. and Melrose, D.B. 1992, Ap. J. 395, 575.

50 Wood, B.E., Brown, A., and Linsky, J.L. 1993a, submitted to ApJ.

[51] Wood, B.E., et al. 1993b, submitted to ApJS.

\section{DYNAMICAL ATMOSPHERES AND WINDS (Lee Anne Willson)}

Advances have occurred in modeling convection in stellar atmospheres; also in modeling the atmospheres of pulsating stars, of stars with dusty winds, and of hot stars with non-spherical winds. These are reviewed below. Other advances reported during the current triennium but not reviewed here include studies of (a) heating of stellar chromospheres and coronae by various types of waves; (b) conditions for the propagation of acoustic and Alfvén waves in the solar and stellar atmospheres; (c) observational constraints on model atmospheres and winds for evolved stars; (d) relativistic outflows in supernovae and from around neutron stars; (e) winds generated in explosive events - novae and supernovae; (e) winds in interacting binary systems; and (f) the detailed hydromagnetic structure of the solar atmosphere and wind. The summaries below also do not include papers that are primarily interpretation of data or modeling for a single star.

Detailed simulations of convective motions in the solar and stellar atmospheres have been carried out and compared with observations by Dravins and Nordlund in a series of papers on "Stellar Granulation" (Dravins 1990, Nordlund and Dravins 1990). Methods for incorporating the dynamical effects of multiperiodic, aperiodic, or stochastic spectra of wave motions, such as those expected to be stimulated by convection or multi-mode pulsation, are discussed in papers by Gail, 
Cuntz, and Ulmschneider (1990) and Koninx and Pijpers (1992).

For many cool giants and supergiants, winds are driven by a combination of the effects of pulsation and radiative forces on grains. It is important to include both pulsation (with a careful treatment of the thermal physics and post-shock nonequilibrium "chemistry) and the effects of dust if the systematics of observed mass loss for AGB stars (Miras) are to be simulated accurately, although for the shorter period, less luminous stars the mass loss process is less dependent on the dust (Bowen and Willson 1991). Sedlmayr and collaborators have been calculating details of the dust formation process in dynamical atmospheres with large excesses of $\mathrm{C} / \mathrm{O}$, and related calculations have been carried out by Dorfi and collaborators (Höfner \& Dorfi 1992). In such atmospheres (which must be rare, as most observed carbon stars have $\mathrm{C} / \mathrm{O}$ close to 1 ), outflows are unstable and may develop shocks even in the absence of pulsation (Fleischer, Gauger and Sedlmayr 1993) possibly leading to a natural explanation for the episodic mass loss observed in R CrB stars (Goeres and Sedlmayr 1992). Netzer and Elitzur (1993) find that for normal chemistry, for both $M$ and $C$ stars, dust driven winds are only possible if $\mathrm{M}>10-7 \mathrm{Ms} / \mathrm{yr}$, and dust- driven winds from carbon stars cannot exceed 10-4 Ms/yr; however, these results may depend critically on their assumption of a single grain size applicable to both types of stars. For the substantially warmer and less luminous RR Lyrae stars, Fokin (1992) has carried out numerical models including synthesis of Ha absorption line profiles; no mass loss occured, but models were not extended far enough out to allow for discovery of mass loss rates below about 10-9 Ms/yr. None of the cited dynamical models has yet incorporated all of the detailed physics that is known to affect the results: details of heating and cooling due to shocks and due to interaction of dust grains with ambient gas; effects of radiative forces on molecules; tracing the complete process of dust grain nucleation and growth; rigorous non-LTE radiative transfer and spectral synthesis; extension of the models far enough out to detect wind formation; and inclusion of both sub-photospheric driving and atmospheric response (including damping of the pulsation). However, models are increasingly including several or even most of these effects. Thus this remains an active area of investigation, with a wealth of observational data waiting to be interpreted more rigorously.

For hot stars, key papers concerning the 2D and 3D structure of the atmospheres and wind appeared during 1990-93. Bjorkman and Cassinelli (1993) find that equatorial disks form naturally as a result of wind driving in the presence of rotation if $\mathrm{v} / \mathrm{vcrit}>.9$ for $\mathrm{O}$ stars and $>.5$ to .6 for $\mathrm{B} 2$ stars, where the effect is maximal. Chen, Marlborough and Waters (1992) inverted observed $v(r)$ to derive constraints on the form of any force(s) besides gravity, rotation, and gas pressure gradient that might be present. Mazzali (1990) explored effects of stellar rotation on the P Cygni line profiles. Kudritzki (1992) included both microturbulance and transsonic outflow in computing weak lines for $\mathrm{O}$ stars. Boyd and Marlborough (1991) computed polarization for rotationally distorted, radiation driven stellar winds for B[e] stars, while Taylor and Cassinelli (1992) pointed out that "polarization cancellation" could occur in certain cases for WR stars with axisymmetric, two-component winds. Poe, Owocki and Castor (1990) computed non-Sobolev, pure absorption models for the winds and found that these have no uniquely defined steady state. Owocki and Zank (1991) examined the effects of viscosity on such flows, and found that it could stabilize some flows. Hummer and Rybicki (1992) find that the criteria for using Sobolev theory in expanding winds are difficult to meet if transfer takes place mainly in the Voigt wings and partial or complete frequency redistribution is considered. Instabilities in hot star winds were also investigated by Owocki and Rybicki (1991) and Lobel, Achmad, de Jager and Nieuwenhuizen (1992), and Drew (1990) pointed out that apparent contradictions between mass loss rates and velocity laws derived from theoretical models and from $\mathrm{Ha}$ observations could be resolved if such instabilities give rise to density inhomogeneities near the star.

\section{References}

Bjorkman, J. E. and J. P. Cassinelli (1993). Formation Around Rotating Stars Due to Ram Pressure Confinement by the Stellar Wind. Ap. J. 409, 429-449.

Bowen, G. H. and L. A. Willson (1991). From Wind to Superwind: The Evolution of Mass-Loss Rates for Mira Models. Ap. J. 375, 153-L56.

Boyd, C. J. and J. M. Marlborough (1991). Continuum Polarization by Electron Scattering in Rotationally Distorted, Radiation-Driven Stellar Winds: B[e] Supergiants. Ap. J. 369: 191-199.

Chen, H., J. M. Marlborough, and L. B. F. M. Waters (1992). Dynamics of the Envelopes of Be 
Stars in the Equatorial Plane. Ap. J. 384, 605-612.

Dravins, D. (1990). Stellar granulation VI. Four-component models and non-solar-type stars. Astr. Ap. 228, 218-230.

Drew, J. E. (1990). Relation Between Mass-Loss Rate and H-alpha Emission for O Star Winds. Ap. J. 357, (No 2), 573-581.

Fleischer, A. J., A. Gauger, and Sedlmayr. (1992). Circumstellar dust shells around long-period variables. I. Dynamical Models of C-stars Including Dust Formation, Growth and Evaporation. Astr. Ap. 266, 321-339.

Fokin, A. B. (1992). Shock waves and H-alpha profiles in the hydrodynamical model of RR Lyrae'. M. N. R. S. $256,26-36$.

Gail, H.-P., M. Cuntz, and Ulmschneider. (1990). Wave pressure in stellar atmospheres due to shock wave trains. Astr. Ap. 234, 359-365.

Goeres, A. and E. Sedlmayr (1992). "The envelopes of R Coronae Borealis stars I. A physical model of the decline events due to dust formation", A \& A 265, 216-236.

Höfner, S. and E. A. Dorfi (1992). Dust formation in winds of long-period vaariables II. dust energetics, optical properties, chemistry, time scales. Astr. Ap. 265 (No 1), 207-215.

Hummer, D. G. and G. B. Rybicki (1992). The Sobolev approximation for line formation with partial frequency redistribution. $A p . J . \mathbf{3 8 7}, 248-257$.

Koninx, J.-P. M. and F. P. Pijpers (1992). The applicability of the linearized theory of sound-wave driven winds. Astr. Ap. 265, 183-195.

Kudritzki, R. P. (1992). The formation of weak metal lines in the atmospheres of O-stars. The role of microturbulence and transonic matter outflow. Astr. Ap. 266, 395-401.

Lobel, A., L. Achmad, de Jager and Nieuwenhuizen. (1992). On the instability parameters of stellar atmosphers. Astr. Ap. 264, 147-152.

Lucy, L. B. and D. C. Abbott (1993). Multiline transfer and the dynamics of Wolf-Rayet winds. Ap. J. 405, 738-746

Mazzali, P. A. (1990). The effect of stellar rotation on the properties of a radiatively driven stellar wind. Astr. Ap. 238, 191-206.

Netzer, N. and M. Elitzur (1992). The dynamics of stellar outflows dominated by interaction of dust and radiation. $A p . J .410,701-713$.

Nordlund, $\AA$. and D. Dravins (1990). Stellar granulation III. Hydrodynamic model atmospheres. Astr. Ap. 228, 155-183.

Owocki, S. P. and G. B. Rybicki (1990). Instabilities in line-driven stellar winds. V. Effect of an optically thick continuum. Ap. J. 368, 261-263.

Owocki, S. P. and G. P. Zank (1991). The effect of viscosity on steady transonic flow with a nodal solution topology. Ap. J. 368, 491-503.

Poe, C. H., S. P. Owocki, and Castor. (1990). The steady state solutions of radiatively driven stellar winds for a non-Sobolev, pure absorption model. Ap. J. 358, 199-213.

Taylor, M. and J. P. Cassinelli (1992). Polarization cancellation in the two-component winds from Wolf-Rayet stars. Ap. J. 401, 311-316.

Velli, M. (1993). On the propagation of ideal, linear Alfvén waves in radially stratified stellar atmospheres and winds. A \& A 270: 304-314.

\section{DISK AND ENVELOPE EMISSION FROM YOUNG STELLAR OBJECTS (Lee Hartmann)}

The present paradigm for $\mathrm{T}$ Tauri stars is that of a low-mass, pre-main sequence star surrounded by a dusty disk $[11,2]$. The disk accounts for the infrared excesses of these stars, powered by a combination of accretion energy and absorption of light from the central star. Accretion probably also provides the energy source for the blue-ultraviolet continuum emission, either in a narrow boundary layer between the Keplerian disk and the slowly-rotating central star [4,9], or at the base of an accretion column [10]. The mass of a typical T Tauri disk is uncertain; the best estimates suggest masses at least the size of the minimum mass solar nebula $\sim 10^{-2} \mathrm{M}_{\odot}$ [5].

Although the disk model for $\mathrm{T}$ Tauri stars seems fairly firmly grounded, there is at least one aspect of great concern, namely the "spectral index" problem. Defining the spectral index $s=d \log F_{\lambda} / \operatorname{dlog} \lambda$, both steady accretion disks [11] and flat disks heated only by light from the central star $[2,3]$ have 
$s=-4 / 3$. In contrast, the median T Tauri spectral energy distribution has $s \sim-2 / 3$ [9], and a small but significant number of objects have $s \sim 0[3,5,9]$. It has become common practice to assume that the observed infrared radiation arises in a disk, and assign an appropriate temperature distribution to it $T \propto R^{-m}$. Such a disk will then exhibit a spectral index $s=2 / m-4$, so for example the "flat spectrum" sources $s \sim 0$ must then have $m=1 / 2$, rather than the standard $m=3 / 4$. It is then up to theory to try to explain why such a temperature distribution occurs.

So far theory has not really been up to the task, particularly for the "flat spectrum" sources. Gravitational instabilities of azimuthal quantum number $=1$ in relatively massive disks have been suggested $[15,1]$, but given the sensitivity of this instability to the outer boundary condition it is not clear how easily these modes are maintained, nor has it been demonstrated that the dissipation produced produces the required temperature distribution. Indeed, there are concerns that such a mechanism would require an unrealistically large accretion rate in the outer disk [9]. The main problem is that, for a "flat spectrum" source, the implied energy release per logarithmic radius interval is constant. This is difficult to achieve, especially in the outer radii of pre-main sequence disks, which are three to four orders of magnitude larger than the inner radii, and thus the gravitational potential energy per unit mass available at such large distances from the central star is small (unless the disk is very massive, which seems unlikely).

An alternative suggestion [9] is that gaseous disks should be "flared", i.e. concave toward the star, and so could be heated more by irradiation at large distances than a flat disk would be. Irradiation by the central star is an attractive mechanism for tapping the energy liberated deep in the gravitational potential well of the system, and redistributing it to much larger radial distances. However, the flared disk explanation suffers the difficulty that dust is expected to coagulate and settle to the midplane of the disk fairly rapidly. Since dust provides all of the absorptive opacity, the disk photosphere would be flat and no enhancement of heating would occur.

Recent suggestions to solve this problem involve irradiation of another dusty component by the central star. Safier [13] suggested that a dusty wind ejected from the disk could be the source of the infrared radiation. Natta [16] offered a variant of this idea, in which the envelope itself is relatively optically thin, but it can scatter a significant fraction of the light down into the opaque, dense disk; the enhanced temperature in the disk produces the required excess infrared radiation. Whitney \& Hartmann [20] showed that the optical and near-infrared scattered light patterns of some young stellar objects imply extensive surrounding, possibly infalling, dusty envelopes. Some of these objects are "flat spectrum" sources, so that the scattered light observations [19] indicate that extended dusty envelopes as well as disks must be included in any consideration of the infrared emission. The final resolution of the spectral index problem is not yet at hand, but it is a good bet that the answer will involve some sort of dusty envelope.

The success of the disk paradigm for low-mass stars has naturally led to suggestions that disk formation might also be important for higher-mass stars. Several attempts have been made to model the spectral energy distributions of the young Herbig Ae/Be stars. Natta et al. $[17,18]$ have focussed on "flat-spectrum" sources among the Ae/Be objects, modelling them by a combination of "flat-spectrum" disk and a tenuous outer envelope. Given the considerations mentioned above, these results are probably rather tentative, especially since all of these objects are heavily embedded in dust, with large, extensive scattered light envelopes at optical and near-infrared wavelengths.

The analyses of Hillenbrand et al. [8] and Lada \& Adams [12] suggested that many Ae/Be stars do have massive disks, and that these disks accrete rapidly (as much as $\sim 10^{-6}-10^{-5} \mathrm{M}_{\odot} \mathrm{yr}^{-1}$ ). These disks must have inner "holes" of $\sim 10 R_{*}$ to account for the general existence of a "bump" or peak in the infrared emission near $\lambda \sim 3 \mu \mathrm{m}$. These authors suggested that this "hole" may not be real, but may simply be where the dust gets destroyed by the high-temperature radiation fields of the star and disk ( $\mathrm{T} \gtrsim 1600 \mathrm{~K})$.

However, Hartmann et al. [7] pointed out that at the high accretion rates required, even if dust is destroyed the gas in the inner disk will be optically thick, and so no "hole" will appear in the spectral energy distribution. Furthermore, if the material is to accrete onto the star it is necessary to radiate much more energy in some wavelength band than is readily observable. For example, if the "inner edge" of the disk is at $10 R_{*}$, but material is accreting all the way to $1 R_{*}$, then $90 \%$ of the energy liberated is missing. It is difficult to conceive of putting this energy into high-frequency radiation; although such radiation would be difficult to observe directly because 
of the large extinction to these objects, one would expect to observe some line radiation of hightemperature species (He I or He II) in the optical which are not seen. Letting the material pile up at the "inner edge" of the disk does not seem to be acceptable either, given the high accretion rates required to match the observations.

Hartmann et al. [7] suggested that the infrared excess emission might be due to dusty envelopes, either around the optical object or an infrared companion. They suggested that the $3 \mu \mathrm{m}$ bump might be produced preferentially by transiently-heated small dust grains. Such grains have been invoked to explain the relatively high-temperature near-infrared thermal emission observed at large distances from B stars (e.g. [14]). A hard photon $\gtrsim 10 \mathrm{eV}$ can transiently heat a small grain to temperatures well in excess of radiative equilibrium, and some high-temperature emission can result before the grain cools off. This mechanism will only work for high-temperature central stars, and it is suggestive that the $3 \mu \mathrm{m}$ peaks are only seen in the Ae/Be stars, but not in the cooler $\mathrm{T}$ Tauri stars. In addition, some Ae/Be stars exhibit near- to mid-infrared emission bands attributed to PAHs (small clusters of polyaromatic hydrocarbon molecules) [6], supporting an model of a dusty envelope containing small grains for some (but by no means all) objects.

The dusty envelope model for Ae/Be stars is not without its own problems, such as how to account for the dynamical state of the dusty envelope on size scales $\sim 2000-200$ A.U., or whether the small grains can survive disruption by the intense radiation fields of the central stars. However, straightfoward application of the disk model raises comparable, if not worse, difficulties. Disk accretion is an attractive possibility for young, intermediate-mass stars, but this cannot be clearly demonstrated yet.

\section{References}

[1] Adams, F. C., Ruden, S. P., \& Shu, F. H. 1989, ApJ, 347, 959

2 Adams, F. C., Lada, C. J., \& Shu, F. H. 1987, ApJ, 312, 788

3 Adams, F. C., Lada, C. J., \& Shu, F. H. 1988, ApJ, 326, 865

4 Basri, G., \& Bertout, C. 1989, ApJ, 341, 340

5 Beckwith, S., Sargent, A., Chini, R., \& Gusten, R. 1990, AJ, 99, 1024

6 Brooke, T.Y., Tokunaga, A.T., \& Strom, S.E. 1993, AJ, in press

7 Hartmann, L., Kenyon, S.J., \& Calvet, N. 1993, ApJ, 407, 219

8. Hillenbrand, L.A., Strom, S.E., Vrba, F.J., \& Keene, J. 1992, ApJ, 397, 613

9] Kenyon, S. J., \& Hartmann, L. 1987, ApJ, 323, 714

10] Königl, A. 1991, ApJL 370, L39

11 Lynden-Bell \& Pringle 1974, MNRAS, 168, 603

12 Lada, C.J., \& Adams, F.C. 1992, ApJ, 393, 278

13. Safier, P. 1993, ApJ, in press

14. Sellgren, K. 1984, ApJ, 277, 623

15 Shu, F. H., Tremaine, S., Adams, F. C., \& Ruden, S. P. 1990, ApJ, 358, 495

16. Natta, A. 1993, ApJ, in press

17 Natta, A., Palla, F., Butner, H.M., Evans, N.J. II, Harvey, P.M. 1992, ApJ, 391, 805

18 Natta, A., Palla, F., Butner, H.M., Evans, N.J. II, Harvey, P.M. 1993, ApJ, 406, 674

19 Weintraub, D.A., Kastner, J.H., Zuckerman, B., \& Gatley, I. 1992, ApJ, 391, 784

20] Whitney, B. A., \& Hartmann, L. 1993, ApJ, 402, 605

\section{THE ATMOSPHERIC STRUCTURE OF VERY COOL STARS (Takashi Tsuji)}

Most stars eventually evolve to very cool stars (red giants and supergiants, AGB stars), where products of stellar nucleosynthesis are mixed to the stellar surface and returned to interstellar space by large mass loss. On the other hand, very low mass stars appear to be very cool too, and they carry a large fraction of the mass of galaxies, possibly including the so-called dark matter. To clarify all these basic roles played by very cool stars, understanding of the atmospheric structure is crucial, since it provides the important basis for interpreting observations of various kinds.

Modeling the atmospheric structure for very cool stars, however, has been more difficult than for hotter stars, because of the complicated molecular opacities, among others. Recently, however, considerable progress has been achieved and it may be not long before classical model atmospheres 
of very cool stars will be quite sophisticated.

\section{Molecular opacities}

The opacity problem is of fundamental importance in the theory of stellar atmosphere, and especially molecular opacity is important in cool stars. Recent progress on molecular opacities and related topics is well reviewed by two meetings: one is "Workshop on Astrophysical Opacities", Caracas, July 1991 (Proceedings ed. Lynas-Gray et al. 1992) and the other is the 146-th IAU Colloquium on "Molecular Opacities in the Stellar Environment", Copenhagen, May 1993 (Proccedings ed. Jørgensen, 1993). Some of the impressive progress reported in these meetings is as follows. First, ab initio computations of molecular structures are now mature enough to provide accurate line positions and intensities for numerous transitions of astrophysically important molecules (e.g., $\mathrm{H}_{2} \mathrm{O}, \mathrm{HCN}, \mathrm{C}_{3}$ ). This will constitute a counter part of dedicated efforts done for atomic lines by Kurucz. Second, progress in experimental molecular physics also provides such basic data as thermodynamical, intensity, and spectroscopic data. Especially, the Fourier Transform Spectroscopy (FTS), pioneered by P. and J. Connes in the 1960's, is now revolutionizing laboratory molecular spectroscopy and providing important data of high precision for molecules of astrophysical interests. Third, helped by the recent computer technolgy, all these results made it possible to develop detailed line-by-line databases for molecular spectra. There is no doubt that the completion of such a database is of basic importance not only for our understanding of the atmospheric structure of cool stars but also for interpreting observational data of high precision to be obtained by new technology, both on the ground and in space.

\section{Model Atmospheres of Evolved Cool Stars}

Efforts of modeling the atmospheres of very cool stars continued. For example, the effects of sphericity have now been investigated with elaborate opacites both for oxygen-rich (Pletz et al., 1992) and carbon-rich (Jørgensen et al., 1992) giant stars. Now, classical model atmospheres of cool (super)giant stars may soon be regarded as quite sophisticated by the use of more accurate and realistic molecular opacities reflecting the recent progress mentioned above. The next problem is to test how well classical models represent real stellar atmospheres.

[ Also, such a modeling of the stellar photosperes is an important prerequisite for modeling the outer atmospheres of cool evolved stars. For example, a classical issue if the radiative force on molecules could accelerate the mass-loss outflow in cool luminous stars is examined on the basis of such a modeling for the case of cool carbon stars (Jørgensen \& Johnson, 1992). Further, diverse efforts for modeling the outer atmospheres are being done. Just to mention a few examples: formation of molecules and dust in the inner shell of oxygen-rich supergiants by considering the effect of chromospheric UV radiation (Beck et al. 1992), thermal structure of the outer envelope of supergiant stars by considering the cooling due to fine structure line emission (Rodgers and Glassgold, 1991), spectroscopic diagnostic of the inner shell of the dusty carbon star by the use of infrared molecular line profiles (Keady and Ridgway, 1993) etc.]

\section{Red and Brown Dwarfs}

Recent increase of the sensitivity in astrophysical observations made it possible to observe stars at the lower end of the main sequence and, further, possible substellar objects named brown dwarfs. Observers, however, claim that there is no model to explain their observations satisfactorily, and effective temperatures of the coolest $M$ dwarfs estimated by different authors differ by as much as 500K! (e.g., Berriman et al., 1992; Kirkpatrick et al., 1993; Tinney et al., 1993). Also, despite extensive searches by various methods, no definite identification of brown dwarfs can be done yet (e.g., Stevenson, 1991; Burrow and Liebert, 1993). Is something wrong in our basic understanding of such very cool and very low luminosity objects? Clearly, more efforts of modeling are needed in this field, and understanding of the atmospheric structures is an important basis to predicting observable properties of these objects.

\section{References}

Beck H.K.B., Gail H.-P., Henkel R., Sedlmayr E., 1992, Astron. Astrophys., 265, 626 
Berriman G.B., Reid I.N., Leggett S.K., 1992, Astrophys. J., 392, L31

Burrow A., Liebert J., 1993, Rev. Mod. Phys., 65, 301

Jørgensen U.G.,(ed.), 1993, Proccedings of the 146-th IAU Colloquium on "Molecular Opacities in the Stellar Environment", Lecture Notes in Physics, Springer-Verlag, in press

Jørgensen U.G., Johnson H.R., 1992, Astron. Astrophys. 265, 168

Jørgensen U.G., Johnson H.R., Nordlund Å., 1992, Astron. Astrophys. 261, 263

Keady J.J., Ridgway S.T., 1993, Astrophys. J., 406, 199

Kirkpatrick J.D., Kelly D.M., Rieke G.H., Liebert J., Allard F., Wehrse R., 1993, Astrophys. J., 402,643

Lynas-Gray A.E., Mendoza C., Zeippen C.J.,(ed), 1992. Proceedings of the Workshop on Astrophysical Opacities, in Rev. Mexicana Astron. Astrof., vol.23, special issue

Plez B., Brett J.M. Nordlund Å., 1992, Astron. Astrophys. 256, 551

Rogers B., Glassgold A.E., 1991, Astrophys. J., 382, 606

Stevenson D.J., 1991, Ann. Rev. Astron. Astrophys., 29, 163

Tinney C.G., Mould J.R., Reid I.N., 1993, Astron. J., 105, 1045

\section{MULTIDIMENSIONAL RADIATIVE TRANSFER (Rainer Wehrse)}

Radiative transfer in three spatial dimensions (usually abbreviated "3D radiative transfer") is needed in the modeling and spectral analysis of objects that have both optically thick and optically thin parts and that do not exhibit point or axial symmetries. In addition, it turns out that it is often advantageous to use 3D radiative transfer methods also for cylindrical media (see below). The determination of radiation fields in such media is basically a six-dimensional problem: there are three spatial and two angle dimensions and there is the frequency. Usually, all points in the 6D space are coupled: the spatial coordinates by the transport operator, the angles and the frequency by the source function. For moving media there is additional coupling by the transport operator. Due to this high dimensionality, methods that are presently sucessfully used for plane-parallel or spherical media can hardly be generalized, and new methods -usually less accurate and much more time and memory consuming - have to be devised. An exception is the Monte-Carlo method (see below) that can be set up in a very similar way for all the various cases.

Note that multidimensional radiative transfer is required in astronomy e.g for the modeling of dust clouds, inhomogeneous gaseous nebulae, or accretion disks but also e.g. in the evaluation of remote sensing data or in the study of cloud formation in the earth's atmosphere.

For a static three dimensional non-refracting medium the radiative transfer equation is written

$$
\frac{d I_{\nu}}{d \mathrm{~s}}=\chi_{\nu}\left(I_{\nu}-S_{\nu}\right)
$$

( $\mathrm{s}=$ vector in ray direction, $I_{\nu}=$ specific intensity at frequency $\nu, \chi=$ extinction coefficient per unit pathlength, $S_{\nu}=$ source function). In a Cartesian coordinate system it takes the particularly convenient form

$$
\mathrm{n} \cdot \nabla I_{\nu}=\chi_{\nu}\left(I_{\nu}-S_{\nu}\right)
$$

$(\mathbf{n}=\mathbf{s} /|\mathbf{s}|=$ unit vector in ray direction), since here the angles between the ray directions and the coordinate axes are the same everywhere and therefore no derivatives with respect to angle occur. The transfer equation in various other coordinate systems has been derived by Jones and Bayazitoglu (1993). The existence and uniqueness of solutions has recently been shown by Johnson and Pitkäranta (1983) and in a more general way by Führer (1993).

For moving media -as in the $1 \mathrm{D}$ case- it is best in most cases to describe the radiation field in the comoving frame. Even for simple velocity fields the transfer equation becomes very complicated, in particular, derivatives of the specific intensity with respect to frequency and angles have to be added. Except for cases of pure absorption only solutions for accretion disks in which the advection and aberration terms are neglected have been obtained up to now (Baschek et al., 1993).

Since analytical solutions are not available (except for some trivial special cases) equation (1) has to be solved numerically. The following classes of methods have been used: 


\section{Monte-Carlo-methods}

In this method (for details see e.g. Langer et al., 1978) the motion of photons through the medium is simulated. It is highly flexible and easy to program. Essentially all photon-matter interactions and geometries can be incorporated without problem. The disadvantage, as in the $1 \mathrm{D}$ case, is the low accuracy, in particular for very optically thin and very optically thick regions in spite of recent improvements in the convergence behavior (cf. Pozdnyakov et al., 1983).

\section{Ray-tracing or characteristics methods}

This method has been used e.g. by Efstathiou and Rowan-Robinson (1990), Steffen and Freitag (1991), Nordlund and others. It is very accurate in principle since it is based on the formal solution of the $1 \mathrm{D}$ problem. In practice, however, its performance is reduced by an extremely high number of necessary interpolations since the values of the absorption and scattering coefficients as well as of the source function are usually given on a prescribed grid whereas the rays pass between the grid points. In addition, the calculation of the source function in the case of high scattering fraction is a problem as it has been for grid methods.

\section{Grid methods}

In these methods either finite differences (Myneni et al., 1990; Stenholm et al., 1991) or finite elements (Turek, 1993, Turek and Wehrse, 1993) are used to convert the partial integro-differential equation (1) in a (usually very large) system of linear algebraic equations that is subsequently solved by iteration. The method recently developed by Turek seems presently to be the most sophisticated since (i) by upwinding it ensures stability, (ii) by using unstructured grids it can well adapt to the specific situation, (iii) by employing non-conforming finite elements the accuracy is further improved, and (iv) by utilizing preconditioning and a modern variant of the conjugate gradient method for the solution of the final linear system of equations even for very large scattering fractions a very good convergence rate can be achieved. In addition, a maximal absolute difference between the solution of the continuous and the discretized problem less than a prescribed number can be guaranteed.

In addition to these methods in simple cases (where e.g. only very few angle grid points are required and in one coordinate the discretisation can be coarse) also the line method (see Schiesser, 1990, for a detailed description of the general method and Ben Jaffel and Vidal-Madjar, 1989, for an implementation) can be applied.

\section{References:}

Baschek, B., Papkalla, R., Wehrse, R. 1993, in: Proc. 2nd. Haifa Conf. on Cataclysmic Variables, Regev, O., Shaviv, G., eds.

Ben Jaffel, L., Vidal-Madjar, A. 1989, Astron. Astrophys. 220, 306

Efstathiou, A., Rowan-Robinson, M. 1990, M.N.R.A.S. 245, 275

Führer, C. 1993, diploma thesis, Inst. f. Appl. Math., Heidelberg University

Johnson, C., Pitkäranta, J. 1983, SIAM J. Num. Anal. 20, 951

Jones, P.D., Bayazitoglu, Y. 1993, J.Q.R.S.T. 48, 427

Langer, S.H., Ross, R.R., McCray, R. 1978, Astrophys. J. 222, 959

Myneni, R.B., Asrar, G., Gerstl, S.A.W. 1990, Transp. Theory St. Phys. 19, 205

Nordlund, A. 1982, Astron. Astrophys. 107, 1

Pozdnyakov, L.A., Sobol', I.M., Sunyaev, R.A. 1983, Sov. Sci. Rev. (Sect. E) 2, 189.

Schiesser, W.E. 1991, The Numerical Method of Lines, Academic Press

Steffen, M., Freytag, B. 1991, Rev. Modern Astron. 4, 43

Stenholm, L.G., Störzer, H., Wehrse, R. 1991, J.Q.S.R.T. 45, 47

Turek, S. 1993, Impact Comp. Sc. Eng., in press

Turek, S., Wehrse, R. 1993, Astron. Astrophys. submitted 